\title{
A combined fit to the Higgs branching ratios at the International Large Detector at ILC
}

\author{
Jonas Kunath ${ }^{a, \dagger, *}$ \\ ${ }^{a}$ Laboratoire Leprince-Ringuet, IN2P3-CNRS, École Polytechnique, Institut Polytechnique de Paris \\ Route de Saclay, 91120 Palaiseau, France \\ E-mail: kunath@llr.in2p3.fr
}

The Higgsstrahlung process as a Higgs boson production mechanism at a lepton collider provides access to a high-purity Higgs boson sample. The Higgs branching ratios can be measured simultaneously by analysing the data inclusively. For this purpose, we divide the sample into classes that distinguish reasonably well between the Higgs decay modes. These class counts are associated to the Higgs branching ratios through a model-independent fit. The fit provides an estimate for each of the Higgs branching ratios with the full matrix of statistical correlations between the channels. These are pure branching ratio measurements, independent of any Higgs production mode cross section measurement.

We present a study on data simulated for the ILD concept at the International Linear Collider (ILC) at $250 \mathrm{GeV}$ center-of-mass energy.

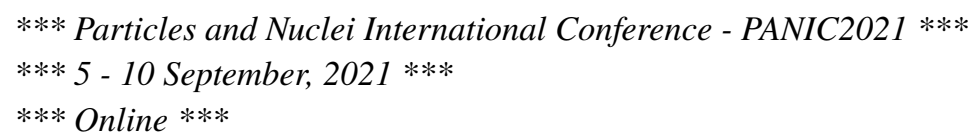

*Speaker

${ }^{\dagger}$ On behalf of the ILD concept group. 


\section{Introduction}

The International Linear Collider (ILC) will initially collide polarized beams of electrons and positrons at center-of-mass energy $\sqrt{s}=250 \mathrm{GeV}$ (ILC250). This energy will then be increased up to $\sqrt{s}=500 \mathrm{GeV}$ [1]. The ILD concept group proposes the International Linear Detector [2,3] as a detector for the ILC. Its silicon trackers allow to measure impact parameters to less than $5 \mu \mathrm{m}$ [3]. With the help of a Time Projection Chamber, transverse momenta of charged particles can be measured with a resolution of $\sigma\left(1 / p_{T}\right)=2 \cdot 10^{-5} \mathrm{GeV}^{-1}$ [3]. The highly granular electromagnetic and hadronic calorimeters are based on the concept of Particle Flow for track reconstruction and particle identification [4]. A jet energy resolution of 3-4\% can be achieved [3]. The surrounding coil provides a magnetic field of $3 \mathrm{~T}$.

Here we focus on the Higgs boson production capabilities at the ILC250. It will produce a large number of Higgs boson events. The dominant Higgs production mode is Higgsstrahlung: $e^{+} e^{-} \rightarrow Z H$. The background rates are low compared to hadron colliders.

One of the improvements compared to a previously presented version of the study [5] is the inclusion of the Standard Model (SM) background processes in the result.

\section{Event selection}
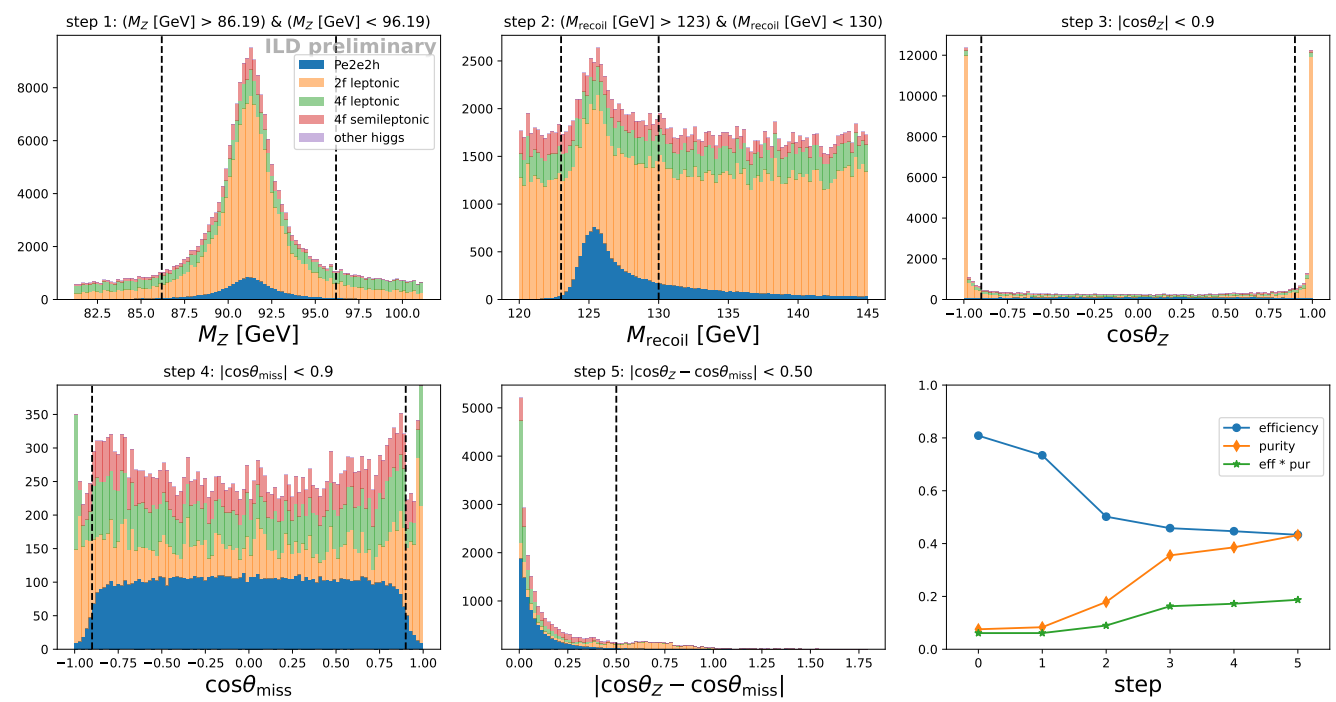

Figure 1: Cut flow for the event selection in the $Z \rightarrow \mu^{+} \mu^{-}$sample. The last figure shows the efficiency and purity after each step.

We target Higgsstrahlung events in which the primary $\mathrm{Z}$ boson decays leptonically, $Z \rightarrow e^{+} e^{-}$ or $Z \rightarrow \mu^{+} \mu^{-}$. Event variables can be reconstructed after the isolated lepton pair is identified and combined with FSR photon candidates. Figure 1 shows the distribution of signal and background for $Z \rightarrow \mu^{+} \mu^{-}$for the variables that were built from the primary $Z$ boson's decay products. Lepton colliders have the advantage that the initial state kinematics is known. This is used when defining the recoil mass: $M_{\text {recoil }}^{2}=s+M_{Z}^{2}-2 \sqrt{s} \cdot E_{Z}$. For signal events, the distribution peaks at $M_{\text {recoil }}=M_{\text {Higgs }}$. 
The cuts presented in Figure 1 lead to a sample with about 50\% purity at 50\% signal efficiency. By definition, the efficiency is constant over Higgs decay modes and the resulting sample is thus unbiased.

We will now build a binned distribution of expected counts from the sample, as shown in Figure 2. Each bin contains the events that fall into a specific class. For the shown bin counts, Standard Model branching ratios are assumed. The black dots additionally contain the background contribution to the $Z \rightarrow \mu^{+} \mu^{-}$Higgsstrahlung sample after event selection. The class definitions can be found in the appendix of [5]. They were chosen so that they distinguish relatively well between the considered Higgs decay modes. The last bin contains all events that do not match any class definition. The relative sensitivity to a Higgs decay mode varies between bins. Next, we will present a fit that uses this variation in sensitivity to derive the Higgs branching ratios (BRs).

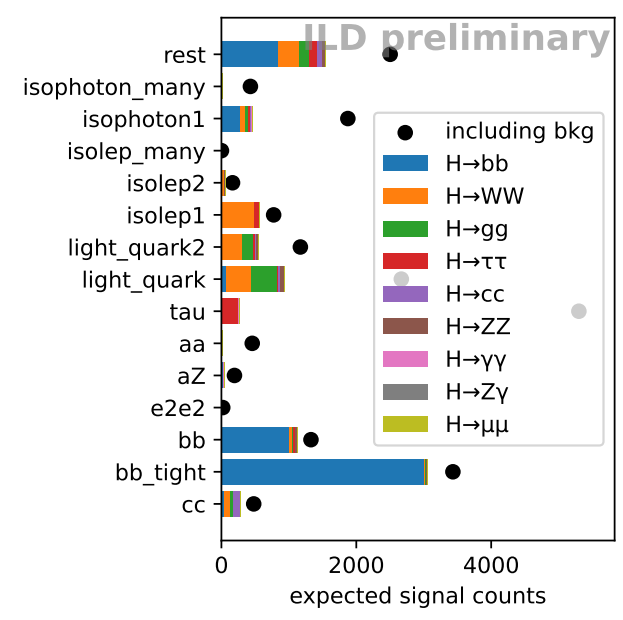

Figure 2: Expected contributions per class from each of the Higgs decay modes.

\section{Branching ratio uncertainties}

\subsection{Setting up the fit}
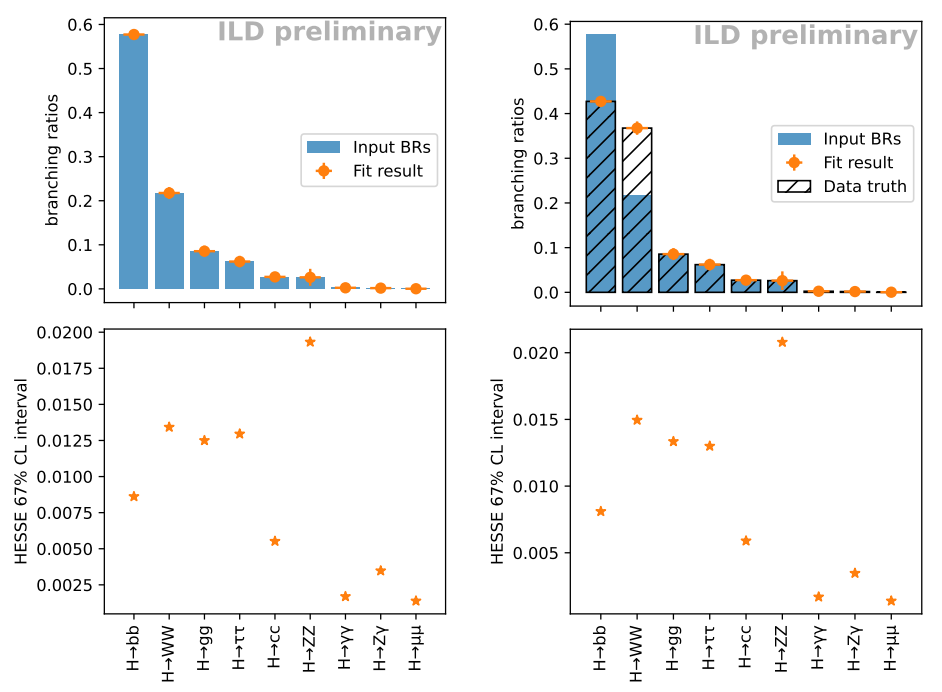

\begin{tabular}{lrr}
\hline & SM BR & $\sigma_{\text {stat }}$ \\
\hline$H \rightarrow b b$ & 57.72 & 0.86 \\
$H \rightarrow W W$ & 21.76 & 1.34 \\
$H \rightarrow g g$ & 8.55 & 1.25 \\
$H \rightarrow \tau \tau$ & 6.20 & 1.30 \\
$H \rightarrow c c$ & 2.72 & 0.55 \\
$H \rightarrow Z Z$ & 2.62 & 1.93 \\
$H \rightarrow \gamma \gamma$ & 0.24 & 0.17 \\
$H \rightarrow Z \gamma$ & 0.17 & 0.35 \\
$H \rightarrow \mu \mu$ & 0.03 & 0.14 \\
\hline
\end{tabular}

Figure 3: Top row: In blue, the BRs that were used in the simulation. The optimum of the fit and its uncertainty are indicated as orange error bars. The starting values of the fit are always the Standard Model Higgs BRs. The considered scenarios are the SM BRs (left) and a BSM scenario described in the text. For the BSM scenario, the BRs that were injected in the data sample are labeled as Data truth.

Bottom row: The absolute statistical uncertainties of the fit per branching ratio.

Table: Preliminary results of a MINUIT fit to the expected event counts. The table gives the fitted values of the Higgs BRs and their absolute statistical uncertainties. All numbers are in percentages. 
The class probabilities for each Higgs decay mode or background process can be determined using simulated events. They are combined into a matrix, where each column corresponds to one process. Applying the truth vector of the process counts to this matrix yields the class counts. Conversely, given the class counts, a fit can be performed to gain access to the process counts. Only the relative contribution of each Higgs BR is taken as a free parameter. With the nine BRs considered in the current analysis, the fit provides the prediction for eight free parameters and the corresponding $8 \times 8$ covariance matrix. They can be converted into mean values and uncertainties for each of the BRs.

\subsection{Results}

In the following we assume a dataset collected at the left-polarized ( $80 \%$ left-polarized electron beam, $30 \%$ right-polarized positron beam) ILC250 with $2000 \mathrm{fb}^{-1}$ of integrated luminosity. Figure 3 gives the resulting values for a combination of the $Z \rightarrow e^{+} e^{-}$and $Z \rightarrow \mu^{+} \mu^{-}$primary $\mathrm{Z}$ boson channels. The left panel of Figure 3 shows the result in the SM case. The central panel supports the claim that the method is truly model independent. Here we assume a model with $B R(H \rightarrow b \bar{b})$ decreased by $15 \%$, and $B R\left(H \rightarrow W^{+} W^{-}\right)$increased by $15 \%$. While this is an unrealistically large deviation from the SM, this shows that the procedure still works far away from the SM hypothesis.

The (absolute) $1 \sigma$-uncertainties are listed in the table on the right side of Figure 3. These values assume BRs close to the SM expectation. Additional non-SM Higgs decays ( $H \rightarrow$ invisible $\left.H \rightarrow \mu^{+} \tau^{-}, H \rightarrow b \bar{c}, \ldots\right)$ could be added to obtain explicit upper limits for these processes.

\section{Conclusion}

The Higgsstrahlung process at a lepton collider allows the measurement of all Higgs branching ratios (BRs) at once within the same unbiased sample. At a Higgs factory such as the ILC250, the method presented in this contribution can be applied to perform precision measurements of the observed BRs and to obtain upper limits for additional Higgs decays. It is a pure BR measurement, independent of any production cross section measurement. The results can be combined with any analysis that does not use the $Z H \rightarrow\left(e^{+} e^{-}, \mu^{+} \mu^{-}\right)$channels.

\section{References}

[1] Linear Collider collaboration, The International Linear Collider Machine Staging Report 2017, 1711.00568.

[2] H. Abramowicz et al., The International Linear Collider Technical Design Report - Volume 4: Detectors, 1306.6329.

[3] ILD Concept Group collaboration, International Large Detector: Interim Design Report, 2003.01116.

[4] J.-C. Brient and H. Videau, The Calorimetry at the future $e^{+} e^{-}$linear collider, eConf C010630 (2001) E3047 [hep-ex/0202004].

[5] J. Kunath et al., A combined fit to the Higgs Branching Ratios at ILD, in International Workshop on Future Linear Colliders, 5, 2021 [2105. 05718]. 